\title{
Necrosis de ciego como causa de abdomen agudo en una paciente con insuficiencia renal crónica
}

\author{
Cecum necrosis as acute abdomen in a patient with renal failure
}

Juan de Dios Díaz-Rosales, ${ }^{*}$ Cecilio R Salva, ${ }^{\ddagger}$ Alma A Rivera-Córdova ${ }^{\ddagger}$

Palabras clave: Apendicitis, colitis isquémica, insuficiencia renal, abdomen agudo.

Keywords: Appendicitis, ischemic colitis, renal insufficiency, abdomen acute.

${ }^{*}$ Cirujano General. Maestro en Ciencias de la Salud, Departamento de Ciencias Médicas. Universidad Autónoma de Ciudad Juárez. Hospital General de Zona No. 35. ${ }^{\ddagger}$ Residente de primer año de Cirugía General. Hospital General de Zona No. 6.

Instituto Mexicano del Seguro Social.

Recibido: 26/11/2018 Aceptado: 14/05/2019

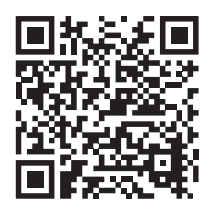

\section{RESUMEN}

La necrosis de ciego es una entidad poco frecuente de etiología incierta, con factores de riesgo bien identificados. Se describe el caso de una paciente diabética (con falla renal crónica en tratamiento con hemodiálisis) con un cuadro de dolor abdominal y sospecha de apendicitis complicada, el diagnóstico definitivo de necrosis de ciego se realizó en el transoperatorio. Se deberá tener en mente la necrosis de ciego dentro del diagnóstico diferencial de abdomen agudo con foco en fosa iliaca derecha en el paciente con comorbilidades como la falla renal.

\section{ABSTRACT}

Cecal necrosis is a rare disease of uncertain etiology with identifiable risk factors. This report describes a case of a female patient with acute abdomen (with comorbidities as type 2 diabetes and renal failure treated with hemodialysis) diagnosed as complicated appendicitis, but real diagnosis was made during surgery as necrosis of cecum. This diagnosis should be considered in patients with acute abdomen focused in right lower quadrant and associated comorbidities as renal failure.

\section{INTRODUCCIÓN}

La isquemia parcial del ciego es una variante infrecuente de colitis isquémica no oclusiva, con una etiología oscura que se asocia a enfermedades crónicas concomitantes como insuficiencia cardiaca ${ }^{1}$ y/o renal (bajo tratamiento con hemodiálisis). ${ }^{2,3}$ Existen otras variantes de colitis isquémica en relación a oclusión vascular (arterial o venosa) aguda, crónica o mixta ${ }^{4}$ y asociada al uso de cocaína. ${ }^{5}$

La colitis isquémica no oclusiva asociada a hemodiálisis (como en el presente caso) se debe a una combinación de varios factores. ${ }^{6}$ Los pacientes con diabetes mellitus, insuficiencia renal crónica y sometidos a tratamiento con hemodiálisis tienen mayor prevalencia de microangiopatía oclusiva y son más susceptibles de cambios hemodinámicos durante sus sesiones de hemodiálisis (ambos factores se suponen como etiología de la necrosis segmentaria del colon), ${ }^{7}$ incluso se ha discutido una sobreinfección bacteriana ${ }^{8}$ y/o micótica ${ }^{9}$ en el colon como cofactores agravantes.

Por lo general, la colitis isquémica se autolimita e incluso puede pasar inadvertida; sin embargo, los casos con necrosis y micro- o macroperforación presentan un cuadro de abdomen agudo muy similar a la presentación de apendicitis. Estos casos deberán ser intervenidos debido a su mortalidad que oscila de 58 a $88 \% .^{3}$

El abordaje y tratamiento quirúrgico puede ser abierto o laparoscópico cuando se tenga la disponibilidad del recurso y las habilidades técnicas. Siempre podrá considerarse la posibilidad de convertir un procedimiento laparoscópico a abierto para el confort del cirujano y la seguridad del paciente. ${ }^{10}$

El objetivo de este reporte es enfatizar una de las causas menos comunes de abdomen agudo en el cuadrante inferior derecho.

Citar como: Díaz-Rosales JD, Salva CR, Rivera-Córdova AA. Necrosis de ciego como causa de abdomen agudo en una paciente con insuficiencia renal crónica. Cir Gen. 2020; 42 (3): 228-231. https://dx.doi.org/10.35366/99965 
Se presenta el caso de una paciente con múltiples comorbilidades y un cuadro de abdomen agudo que simuló una apendicitis complicada.

\section{PRESENTACIÓN DEL CASO}

Paciente femenino de 66 años, con antecedente de diabetes tipo 2 de larga evolución (33 años), retinopatía e insuficiencia renal crónica en tratamiento con hemodiálisis, además de hipertensión arterial descontrolada y dislipidemia. Se presentó al Servicio de Urgencias con dolor abdominal (lo describe como opresivo) de 24 horas de evolución, que inició en mesogastrio e irradió hacia la fosa iliaca derecha (FID), con aumento de intensidad hasta ser incapacitante. A la exploración física con McBurney, Rovsing, Dunphy y puño-talo percusión positivos. Además, presentó distensión abdominal, náusea y vómito. Llamó la atención la presencia de evacuaciones líquidas en cinco ocasiones (sin moco o sangre).

Los laboratorios a su ingreso: glucosa 316 $\mathrm{mg} / \mathrm{dl}$, sodio $130 \mathrm{mEq} / \mathrm{l}$, potasio $5 \mathrm{mEq} / \mathrm{l}$, cloro $95 \mathrm{mEq} / \mathrm{l}$, creatinina $8 \mathrm{mg} / \mathrm{dl}$, nitrógeno ureico sanguíneo $46.9 \mathrm{mg} / \mathrm{dl}$, urea $100.1 \mathrm{mg} /$ $\mathrm{dl}$, hemoglobina $8 \mathrm{~g} / \mathrm{dl}$, hematocrito $25 \%$, leucocitosis de $18.2 \times 10^{9} /$, neutrófilos $87 \%$, plaquetas $163 \times 10^{9} /$, tiempo de protrombina 16 segundos, INR 1.27 y TTP de 29.6 segundos.
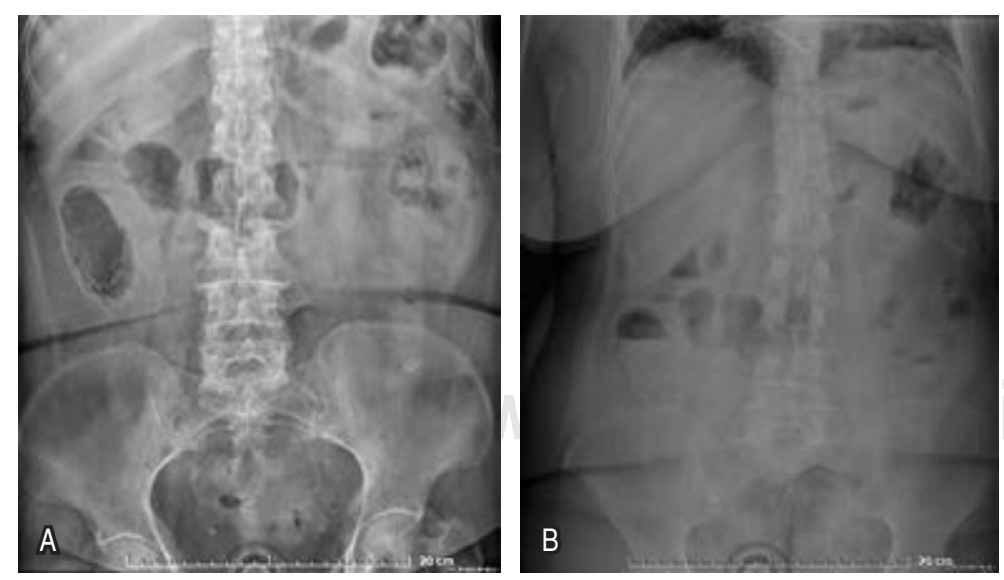

Figura 1: A) Se observa asa centinela y neumatosis intestinal en hemiabdomen derecho. B) Se observa la misma asa centinela con un nivel hidroaéreo y neumatosis intestinal.
La radiografía de abdomen (en decúbito y en bipedestación) mostró asa centinela y un nivel hidroaéreo en el cuadrante inferior derecho (CID) (Figura 1). Se realizó un diagnóstico clínico (puntuación de Alvarado 8) de apendicitis y se procedió a realizar una apendicectomía por laparotomía exploradora. Se realizó una incisión media infraumbilical, al llegar a cavidad se corroboró inflamación intestinal de la región ileal distal, en ciego y colon ascendente. Al levantar el ciego se encontró una necrosis de espesor total y de extensión parcial $(6 \times 8$ $\mathrm{cm})$ a nivel del borde antimesentérico. Dicha necrosis tenía los bordes bien delimitados, mientras que el resto del ciego y apéndice se encontraban con adecuada perfusión, sin datos macroscópicos de sufrimiento isquémico, no hubo otros hallazgos importantes (Figura 2). Se procedió a resecar el segmento de íleon distal $(5 \mathrm{~cm})$ y el ciego en su totalidad, cerrando el colon ascendente en bolsa de Hartmann (dos planos, con poliglactina 910 de 2-0), dejando una ileostomía terminal.

La paciente cursó con un postquirúrgico tórpido, con exacerbación de la falla renal y colapso cardiorrespiratorio dentro de las siguientes 72 horas, falleciendo debido a esta causa.

\section{DISCUSIÓN}

La colitis isquémico-necrótica aislada de ciego es una patología poco frecuente, asociada a enfermedades crónicas como la falla cardiaca ${ }^{1} \mathrm{y} / \mathrm{o}$ renal..$^{2,3}$ Aunque no existe un estudio "estándar de oro", la tomografía puede revelar datos que sugieren el diagnóstico como aumento de volumen en la pared del ciego, neumatosis intestinal y/o líquido libre en la cavidad. ${ }^{11}$

Aunque la mayoría de los autores sugieren la realización de una hemicolectomía (por el riesgo de sufrimiento en el resto de colon) con ileostomía derivativa o en casos seleccionados con anastomosis primaria (íleo-transverso anastomosis), ${ }^{12}$ la decisión de realizar sólo la resección del tejido involucrado se basó en el pobre estado de la paciente, el hallazgo inesperado y en que la sola resección del ciego involucrado (con ileostomía derivativa) es una opción (sin mayores complicaciones) descrita en la literatura. ${ }^{13,14}$ 

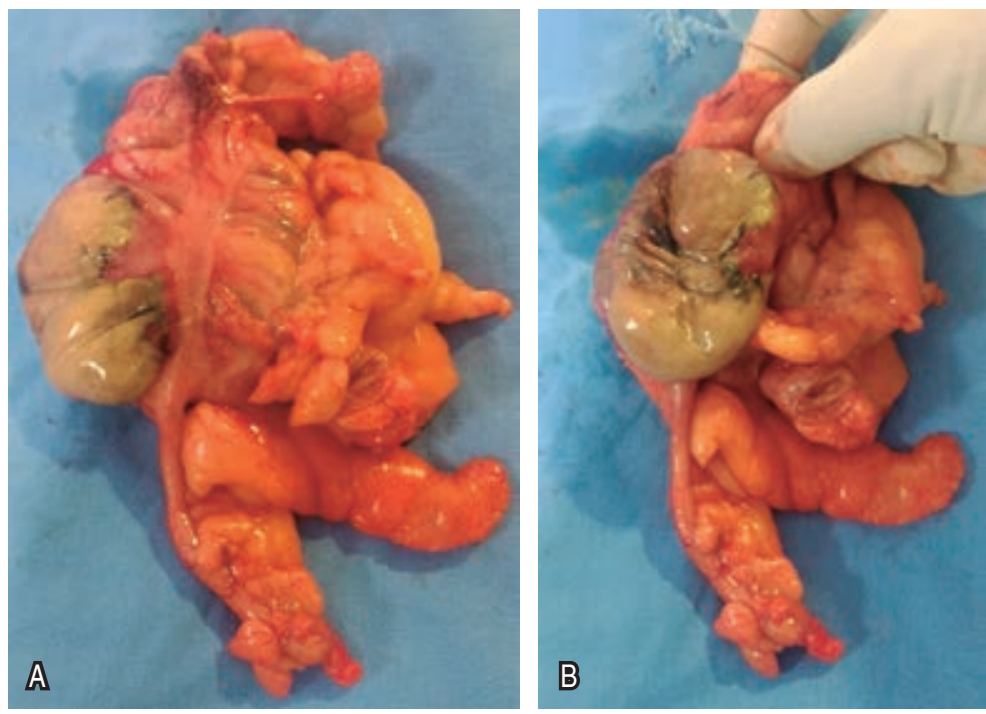

Figura 2: A) Se observa el segmento de colon (ciego) ileon distal, mesoapéndice inflamado y apéndice cecal sin datos de afección. B) La necrosis del ciego en su cara antimesentérica con bordes bien delimitados cercanos a la tenía cecal.

La mortalidad en estos pacientes posiblemente no se debe a la propia necrosis del segmento del colon, sino a la avanzada edad de éstos y a la exacerbación de sus problemas cardiacos y/o renales crónicos por el estrés quirúrgico que produce el tratamiento de urgencia. $^{15}$

La posibilidad de una necrosis de ciego en el escenario de un abdomen agudo con foco en cuadrante inferior derecho siempre deberá tenerse en mente (en pacientes con factores de riesgo como cardiopatía y/o nefropatía en hemodiálisis). Sin embargo, otras patologías infrecuentes como divertículo de ciego, ${ }^{16}$ perforación cecal, ${ }^{17}$ invaginación intestinal ${ }^{18}$ y apendicitis epiploica ${ }^{19}$ (entre otras) pueden también ser la causa del cuadro clínico en cuestión.

\section{CONCLUSIÓN}

El diagnóstico diferencial de abdomen agudo con foco en la FID es diverso, siempre se deberá pensar en las causas más comunes como la apendicitis; sin embargo, se deberán tener en mente aquellos diagnósticos menos frecuentes en pacientes con enfermedades crónicas.

\section{REFERENCIAS}

1. Nessar G, Kucukaksu S, Zengin NI, Tasdemir O, Kayaalp C. Ischemic necrosis of the right colon in a patient with a ventricular assist device system. Tech Coloproctol. 2007; 11: 275-277.

2. Schuler JG, Hudlin MM. Cecal necrosis: Infrequent variant of ischemic colitis. Dis Colon Rectum. 2000; 43: 708-712.

3. Cakar E, Ersoz F, Bag M, Bayrak S, Colak S, Bektas H, et al. Isolated cecal necrosis: our surgical experience and a review of the literature. Turkish J Surg. 2014; 30: 214-218.

4. Watanabe T, Tomita S, Shirane H, Okabe Y, Orino A, Todo A, et al. Cecal necrosis due to ischemic colitis mimicking an abscess on sonography. J Ultrasound Med. 2006; 25: 393-396.

5. Flores-Salazar LO, González-Tovar J, MuñozMaldonado GE, Delgado-Morquecho M. Perforación de ciego por colitis isquémica asociada a uso de cocaína. Reporte de caso. Rev Chil Cir. 2017; 70.

6. Flobert C, Cellier C, Berger A, Ngo A, Cuillerier E, Landi $B$, et al. Right colonic involvement is associated with severe forms of ischemic colitis and occurs frequently in patients with chronic renal failure requiring hemodialysis. Am J Gastroenterol. 2000; 95: 195-198.

7. Reyes-Zamorano J. Necrosis y estenosis por colitis isquémica no oclusiva. Reporte de dos casos y revisión de la bibliografía. Cir Cir. 2014; 82: 442-447.

8. Wiesner W, Mortelé KJ, Glickman JN, Ros PR. "Cecal gangrene": a rare cause of right-sided inferior abdominal quadrant pain, fever, and leukocytosis. Emerg Radiol. 2002; 9: 292-295.

9. Eyvaz K, Sikar HE, Gokceimam M, Kucuk HF, Kurt N. A rare cause of acute abdomen: isolated necrosis of the cecum. Turkish J Surg. 2018; 11: 1-3.

10. Karabay O, Genco Erdem M, Hasbahceci M. Partial cecum necrosis as a rare cause of acute abdominal pain in an elderly patient. J Coll Physicians Surg Pak. 2018; 28: S81-S83.

11. Shahverdi E, Morshedi M, Oraei-Abbasian F, Allahverdi Khani M, Khodayarnejad R. A rare case of vasculitis patched necrosis of cecum due to Behçet's disease. Case rep surg. 2017; 2017: 1693737.

12. Hunter JP, Saratzis A, Zayyan K. Spontaneous, isolated caecal necrosis: report of a case, review of the literature, and updated classification. Acta Chir Belg. 2013; 113: 60-63.

13. Dirican A, Unal B, Bassulu N, Tatlı F, Aydin C, Kayaalp C. Isolated cecal necrosis mimicking acute appendicitis: a case series. J Med Case Rep. 2009; 3: 7443.

14. Perko Z, Bilan K, Vilovic K, Druzijanić N, Kraljević $D$, Juriicić J, et al. Partial cecal necrosis treated by laparoscopic partial cecal resection. Coll Antropol. 2006; 30: 937-939.

15. Gundes E, Kucukkartallar T, Çolak MH, Cakir M, Aksoy F. Ischemic necrosis of the cecum: a single center experience. Korean J Gastroenterol. 2013; 61: 265269.

16. Nemeth K, Vaughan S. Cecal diverticulitis: a diagnostic conundrum. West J Emerg Med. 2015; 16: 316-317.

17. Kaushal-Deep SM, Anees A, Khan S, Khan MA, Lodhi M. Primary cecal pathologies presenting as 
acute abdomen and critical appraisal of their current management strategies in emergency settings with review of literature. Int J Crit IIIn Inj Sci. 2018; 8: 90-99.

18. Díaz-Rosales JD, Enriquez-Dominguez L, Castillo-Moreno JR, Gutierrez-Ramirez PG. Adult intussusception by tumor in ileum: a diagnostic dilemma. Int J Students Res. 2012; 2: 18-20.

19. Molinares Arévalo B, Castrillón GA, Restrepo R. Apendicitis epiploica. Reporte de cuatro casos. Rev Colomb Cir. 2006; 21: 196-200.

Consideraciones y responsabilidad ética: Privacidad de los datos. De acuerdo a los protocolos establecidos en el centro de trabajo de los autores, estos declaran que han seguido los protocolos sobre la privacidad de datos de pacientes preservado su anonimato.

Financiamiento: No se recibió apoyo financiero para la realización de este trabajo.

Conflicto de intereses: Los autores declaran que no existe ningún conflicto de intereses en la realización del trabajo. 